\title{
The Relation between the Stabilization Problem for Discrete Event Systems Modeled with Timed Petri Nets via Lyapunov Methods and Max-Plus Algebra
}

\author{
Zvi Retchkiman Konigsberg \\ Instituto Politécnico Nacional, CIC Mineria 17-2, Col. Escandon, Mexico D.F 11800, Mexico \\ Email: $\underline{\text { mzvi@cic.ipn.mx }}$
}

Received 19 April 2015; accepted 7 July 2015; published 14 July 2015

\begin{abstract}
A discrete event system is a dynamical system whose state evolves in time by the occurrence of events at possibly irregular time intervals. Timed Petri nets are a graphical and mathematical modeling tool applicable to discrete event systems in order to represent its states evolution where the timing at which the state changes is taken into consideration. One of the most important performance issues to be considered in a discrete event system is its stability. Lyapunov theory provides the required tools needed to aboard the stability and stabilization problems for discrete event systems modeled with timed Petri nets whose mathematical model is given in terms of difference equations. By proving stability one guarantees a bound on the discrete event systems state dynamics. When the system is unstable, a sufficient condition to stabilize the system is given. It is shown that it is possible to restrict the discrete event systems state space in such a way that boundedness is achieved. However, the restriction is not numerically precisely known. This inconvenience is overcome by considering a specific recurrence equation, in the max-plus algebra, which is assigned to the timed Petri net graphical model.
\end{abstract}

\section{Keywords}

Discrete Event Systems, Lyapunov Methods, Max-Plus Algebra, Timed Petri Nets

\section{Introduction}

A discrete event system, is a dynamical system whose state evolves in time by the occurrence of events at possibly irregular time intervals. Timed Petri nets are a graphical and mathematical modeling tool applicable to discrete event systems in order to represent its states evolution where the timing at which the state changes is taken into consideration Timed Petri nets are known to be useful for analyzing the systems properties in addition of being a paradigm for describing and studying information processing systems, where the timing at which the state changes is taken into consideration. For a detailed discussion of Petri net theory see [1] and the references 
quoted therein. One of the most important performance issues to be considered in a discrete event system is its stability. Lyapunov theory provides the required tools needed to aboard the stability and stabilization problems for discrete event systems modeled with timed Petri nets whose mathematical model is given in terms of difference equations [2]. By proving stability one guarantees a bound on the discrete event systems state dynamics. When the system is unstable, a sufficient condition to stabilize the system is given. It is shown that it is possible to restrict the discrete event systems state space in such a way that boundedness is achieved. However, the restriction is not numerically precisely known. This inconvenience is overcome by considering a specific recurrence equation, in the max-plus algebra, which is assigned to the timed Petri net graphical model. This paper proposes a methodology consisting in combining Lyapunov theory with max-plus algebra to give a precise solution to the stabilization problem for discrete event systems modeled with timed Petri nets. The presented methodology results to be innovative and it is not, in general, known. The main objective of the paper is to spread its results along large audiences. The paper is organized as follows. In Section 2, Lyapunov theory for discrete event systems modeled with Petri nets is given. Section 3 presents max-plus algebra and max-plus recurrence equations for timed event Petri nets. Section 4 considers the solution to the stabilization problem for discrete event systems modeled with timed Petri nets. Finally, the paper ends with some conclusions.

\section{Lyapunov Stability and Stabilization of Discrete Event Systems Modeled with Petri Nets [2]-[4]}

NOTATION: $N=\{0,1,2, \ldots\}, R_{+}=[0, \infty), N_{n_{0}}^{+}=\left\{n_{0}, n_{0}+1, \ldots, n_{0}+k, \ldots\right\}, n_{0} \geq 0$. Given $x, y \in R^{n}, \quad x \leq y$ is equivalent to $x_{i} \leq y_{i}, \forall i$. A function $f(n, x), f: N_{n_{0}}^{+} \times R^{n} \rightarrow R^{n}$ is called nondecreasing in $x$ if given $x, y \in R^{n}$ such that $x \geq y$ and $n \in N_{n_{0}}^{+}$then, $f(n, x) \geq f(n, y)$. Consider systems of first ordinary difference equations given by

$$
x(n+1)=f[n, x(n)], x\left(n_{o}\right)=x_{0}, n \in N_{n_{0}}^{+}
$$

where $n \in N_{n_{0}}^{+}, \quad x(n) \in R^{n}$ and $f: N_{n_{0}}^{+} \times R^{n} \rightarrow R^{n}$ is continuous in $x(n)$.

Definition 1 The $n$ vector valued function $\Phi\left(n, n_{0}, x_{0}\right)$ is said to be a solution of (1) if

$\Phi\left(n_{0}, n_{0}, x_{0}\right)=x_{0}$ and $\Phi\left(n+1, n_{0}, x_{0}\right)=f\left(n, \Phi\left(n, n_{0}, x_{0}\right)\right)$ for all $n \in N_{n_{0}}^{+}$.

Definition 2 The system (1) is said to be practically stable, if given $(\lambda, A)$ with $0<\lambda<A$, then

$\left|x_{0}\right|<\lambda \Rightarrow\left|x\left(n, n_{0}, x_{0}\right)\right|<A, \forall n \in N_{n_{0}}^{+}, n_{0} \geq 0$

Definition 3 A continuous function $\alpha:[0, \infty) \rightarrow[0, \infty)$ is said to belong to class $\mathcal{K}$ if $\alpha(0)=0$ and it is strictly increasing.

Consider a vector Lyapunov function $v(n, x(n)), \quad v: N_{n_{0}}^{+} \times R^{n} \rightarrow R_{+}^{p}$ and define the variation of $v$ relative to (1) by

$$
\Delta v=v(n+1, x(n+1))-v(n, x(n))
$$

Theorem 4 Let $v: N_{n_{0}}^{+} \times R^{n} \rightarrow R_{+}^{p}$ be a continuous function in $x$, define the function $v_{0}(n, x(n))=\sum_{i=1}^{p} v_{i}(n, x(n))$ such that satisfies the estimates

$$
b(|x|) \leq v_{0}(n, x(n)) \leq a(|x|) ; a, b \in \mathcal{K}, \Delta v(n, x(n)) \leq w(n, v(n, x(n)))
$$

for $n \in N_{n_{0}}^{+}, \quad x(n) \in R^{n}$, where $w: N_{n_{0}}^{+} \times R_{+}^{p} \rightarrow R^{p}$ is a continuous function in the second argument. Assume that: $g(n, e) \triangleq e+w(n, e)$ is nondecreasing in $e, 0<\lambda<A$ are given and finally that $a(\lambda)<b(A)$ is satisfied. Then, the practical stability properties of

$$
e(n+1)=g(n, e(n)), e\left(n_{0}\right)=e_{0} \geq 0 .
$$

imply the practical stability properties of system (1).

Corollary 5 In Theorem (4): If $w(n, e) \equiv 0$ we get uniform practical stability of (1) which implies structural stability. 
Definition 6 A Petri net is a 5-tuple, $P N=\left\{P, T, F, W, M_{0}\right\}$ where: $P=\left\{p_{1}, p_{2}, \ldots, p_{m}\right\}$ is a finite set of places, $T=\left\{t_{1}, t_{2}, \ldots, t_{n}\right\}$ is a finite set of transitions, $F \subset(P \times T) \cup(T \times P)$ is a set of arcs, $W: F \rightarrow N_{1}^{+}$is a weight function, $M_{0}: P \rightarrow N$ is the initial marking, $P \cap T=\varnothing$ and $P \cup T \neq \varnothing$.

Definition 7 The clock structure associated with a place $p_{i} \in P$ is a set $\mathbf{V}=\left\{V_{i}: p_{i} \in P\right\}$ of clock sequences $V_{i}=\left\{v_{i, 1}, v_{i, 2}, \ldots\right\}, \quad v_{i, k} \in R^{+}, \quad k=1,2, \ldots$

The positive number $v_{i, k}$, associated to $p_{i} \in P$, called holding time, represents the time that a token must spend in this place until its outputs enabled transitions $t_{i, 1}, t_{i, 2}, \ldots$, fire. We partition $P$ into subsets $P_{0}$ and $P_{h}$, where $P_{0}$ is the set of places with zero holding time, and $P_{h}$ is the set of places that have some holding time.

Definition 8 A timed Petri net is a 6-tuple $T P N=\left\{P, T, F, W, M_{0}, \mathbf{V}\right\}$ where $\left\{P, T, F, W, M_{0}\right\}$ are as before, and $\mathbf{V}=\left\{V_{i}: p_{i} \in P\right\}$ is a clock structure. A timed Petri net is a timed event petri net when every $p_{i} \in P$ has one input and one output transition, in which case the associated clock structure set of a place $p_{i} \in P$ reduces to one element $V_{i}=\left\{v_{i}\right\}$.

Notice that if $W(p, t)=\alpha$ (or $W(t, p)=\beta$ ) then, this is often represented graphically by $\alpha,(\beta)$ arcs from $p$ to $t$ ( $t$ to $p$ ) each with no numeric label.

Let $M_{k}\left(p_{i}\right)$ denote the marking (i.e., the number of tokens) at place $p_{i} \in P$ at time $k$ and let $M_{k}=\left[M_{k}\left(p_{1}\right), \ldots, M_{k}\left(p_{m}\right)\right]^{T}$ denote the marking (state) of $P N$ at time $k$. A transition $t_{j} \in T$ is said to be enabled at time $k$ if $M_{k}\left(p_{i}\right) \geq W\left(p_{i}, t_{j}\right)$ for all $p_{i} \in P$ such that $\left(p_{i}, t_{j}\right) \in F$. It is assumed that at each time $k$ there exists at least one transition to fire. If a transition is enabled then, it can fire. If an enabled transition $t_{j} \in T$ fires at time $k$ then, the next marking for $p_{i} \in P$ is given by

$$
M_{k+1}\left(p_{i}\right)=M_{k}\left(p_{i}\right)+W\left(t_{j}, p_{i}\right)-W\left(p_{i}, t_{j}\right) .
$$

Let $A=\left[a_{i j}\right]$ denote an $n \times m$ matrix of integers (the incidence matrix) where $a_{i j}=a_{i j}^{+}-a_{i j}^{-}$with $a_{i j}^{+}=W\left(t_{i}, p_{j}\right)$ and $a_{i j}^{-}=W\left(p_{j}, t_{i}\right)$. Let $u_{k} \in\{0,1\}^{n}$ denote a firing vector where if $t_{j} \in T$ is fired then, its corresponding firing vector is $u_{k}=[0, \ldots, 0,1,0, \ldots, 0]^{T}$ with the one in the $j^{\text {th }}$ position in the vector and zeros everywhere else. The nonlinear difference matrix equation describing the dynamical behavior represented by a $P N$ is:

$$
M_{k+1}=M_{k}+A^{T} u_{k}
$$

where if at step $k, a_{i j}^{-}<M_{k}\left(p_{j}\right)$ for all $p_{i} \in P$ then, $t_{i} \in T$ is enabled and if this $t_{i} \in T$ fires then, its corresponding firing vector $u_{k}$ is utilized in the difference equation to generate the next step. Notice that if $M$ can be reached from some other marking $M$ and, if we fire some sequence of $d$ transitions with corresponding firing vectors $u_{0}, u_{1}, \ldots, u_{d-1}$ we obtain that

$$
M^{\prime}=M+A^{T} u, u=\sum_{k=0}^{d-1} u_{k} .
$$

Let $\left(N_{n_{0}}^{m}, d\right)$ be a metric space where $d: N_{n_{0}}^{m} \times N_{n_{0}}^{m} \rightarrow R_{+}$is defined by

$d\left(M_{1}, M_{2}\right)=\sum_{i=1}^{m} \zeta_{i}\left|M_{1}\left(p_{i}\right)-M_{2}\left(p_{i}\right)\right| ; \zeta_{i}>0$ and consider the matrix difference equation which describes the dynamical behavior of the discrete event system modeled by a $P N$, see (7).

Proposition 9 Let $P N$ be a Petri net. $P N$ is uniform practical stable if there exists a $\Phi$ strictly positive $m$ vector such that

$$
\Delta v=u^{T} A \Phi \leq 0
$$

Moreover, $P N$ is uniform practical asymptotic stable if the following equation holds

$$
\Delta v=u^{T} A \Phi \leq-c(e), c \in \mathcal{K}
$$

Lemma 10 Let suppose that Proposition (9) holds then,

$$
\Delta v=u^{T} A \Phi \leq 0 \Leftrightarrow A \Phi \leq 0
$$

Remark 11 Notice that since the state space of a TPN is contained in the state space of the same now not 
timed PN, stability of PN implies stability of the TPN.

\section{Lyapunov Stabilization}

Definition 12 Let $P N$ be a Petri net. $P N$ is said to be stabilizable if there exists a firing transition sequence with transition count vector $u$ such that system (7) remains bounded.

Proposition 13 Let $P N$ be a Petri net. $P N$ is stabilizable if there exists a firing transition sequence with transition count vector $u$ such that the following equation holds

$$
\Delta v=A^{T} u \leq 0
$$

Remark 14 By fixing a particular $u$, which satisfies (11), the state space is restricted to those markings that are finite.

\section{Max-Plus Algebra [5] [6]}

\subsection{Basic Definitions}

NOTATION: $\epsilon=-\infty, e=0, \mathbb{R}_{\max }=\mathbb{R} \cup\{\epsilon\}, \underline{n}=1,2, \ldots, n$. Let $a, b \in \mathbb{R}_{\max }$ and define the operations $\oplus$ and $\otimes$ by: $a \oplus b=\max (a, b)$ and $a \otimes b=a+b$.

Definition 15 The set $\mathbb{R}_{\max }$ with the two operations $\oplus$ and $\otimes$ is called a max-plus algebra and is denoted by $\mathfrak{R}_{\max }=\left(\mathbb{R}_{\max }, \oplus, \otimes, \epsilon, e\right)$.

Definition 16 A semiring is a nonempty set $R$ endowed with two operations $\oplus_{R}$, $\otimes_{R}$, and two elements $\epsilon_{R}$ and $e_{R}$ such that: $\oplus_{R}$ is associative and commutative with zero element $\epsilon_{R}, \otimes_{R}$ is associative, distributes over $\oplus_{R}$, and has unit element $e_{R}, \epsilon_{R}$ is absorbing for $\otimes_{R}$ i.e., $a \otimes_{R} \epsilon=\epsilon_{R} \otimes a=a, \forall a \in R$. In addition if $\otimes_{R}$ is commutative then $R$ is called a commutative semiring, and if $\oplus_{R}$ is such that $a \oplus_{R} a=a, \forall a \in R$ then it is called idempotent.

Theorem 17 The max-plus algebra $\mathfrak{R}_{\max }=\left(\mathbb{R}_{\max }, \oplus, \otimes, \epsilon, e\right)$ has the algebraic structure of a commutative and idempotent semiring.

\subsection{Matrices and Graphs}

Let $\mathbb{R}_{\max }^{n \times n}$ be the set of $n \times n$ matrices with coefficients in $\mathbb{R}_{\max }$ with the following operations: The sum of matrices $A, B \in \mathbb{R}_{\max }^{n \times n}$, denoted $A \oplus B$ is defined by: $(A \oplus B)_{i j}=a_{i j} \oplus b_{i j}=\max \left(a_{i j}, b_{i j}\right)$ for $i$ and $j \in \underline{n}$. The product of matrices $A \in \mathbb{R}_{\max }^{n \times l}, B \in \mathbb{R}_{\max }^{l \times n}$, denoted $A \otimes B$ is defined by: $(A \otimes B)_{i k}=\bigotimes_{j=1}^{l}\left(a_{i j} \otimes b_{j k}\right)$ for $i$ and $k \in \underline{n}$. Let $\mathcal{E} \in \mathbb{R}_{\max }^{n \times n}$ denote the matrix with all its elements equal to $\epsilon$ and denote by $E \in \mathbb{R}_{\max }^{n \times n}$ the matrix which has its diagonal elements equal to $e$ and all the other elements equal to $\epsilon$. Then, the following result can be stated.

Theorem 18 The 5-tuple $\mathfrak{R}_{\max }^{n \times n}=\left(\mathbb{R}_{\max }^{n \times n}, \oplus, \otimes, \mathcal{E}, E\right)$ has the algebraic structure of a noncommutative idempotent semiring.

Definition 19 Let $A \in \mathbb{R}_{\max }^{n \times n}$ and $k \in \mathbb{N}$ then the $k$-th power of $A$ denoted by $A^{\otimes k}$ is defined by: $A^{\otimes k}=A \otimes A \otimes \cdots \otimes A$, ( $k$ times), where $A^{\otimes 0}$ is set equal to $E$.

Definition 20 A matrix $A \in \mathbb{R}_{\max }^{n \times n}$ is said to be regular if $A$ contains at least one element distinct from $\epsilon$ in each row.

Definition 21 Let $\mathcal{N}$ be a finite and non-empty set and consider $\mathcal{D} \subseteq \mathcal{N} \times \mathcal{N}$. The pair $G=(\mathcal{N}, \mathcal{D})$ is called a directed graph, where $\mathcal{N}$ is the set of elements called nodes and $\mathcal{D}$ is the set of ordered pairs of nodes called arcs. A directed graph $G=(\mathcal{N}, \mathcal{D})$ is called a weighted graph if a weight $w(i, j) \in \mathbb{R}$ is associated with any arc $(i, j) \in \mathcal{D}$.

Let $A \in \mathbb{R}_{\max }^{n \times n}$ be any matrix, a graph $\mathcal{G}(A)$, called the communication graph of $A$, can be associated as follows. Define $N(A)=\underline{n}$ and a pair $(i, j) \in \underline{n} \times \underline{n}$ will be a member of $\mathcal{D}(A) \Leftrightarrow a_{j i} \neq \epsilon$, where $\mathcal{D}(A)$ denotes the set of arcs of $\mathcal{G}(A)$.

Definition 22 A path from node $i$ to node $j$ is a sequence of arcs $p=\left\{\left(i_{k}, j_{k}\right) \in \mathcal{D}(A)\right\}_{k \in m}$ such that $i=i_{1}, j_{k}=i_{k+1}$, for $k<m$ and $j_{m}=j$. The path $p$ consists of the nodes $i=i_{1}, i_{2}, \ldots, i_{m}, j_{m}=j$ with length $m$ denoted by $|p|_{1}=m$. In the case when $i=j$ the path is said to be a circuit. A circuit is said to be 
elementary if nodes $i_{k}$ and $i_{l}$ are different for $k \neq l$. A circuit consisting of one arc is called a self-loop.

Let us denote by $P(i, j ; m)$ the set of all paths from node $i$ to node $j$ of length $m \geq 1$ and for any arc $(i, j) \in \mathcal{D}(A)$ let its weight be given by $a_{i j}$ then the weight of a path $p \in P(i, j ; m)$ denoted by $|p|_{w}$ is defined to be the sum of the weights of all the arcs that belong to the path. The average weight of a path $p$ is given by $|p|_{w} /|p|_{1}$. Given two paths, as for example, $p=\left(\left(i_{1}, i_{2}\right),\left(i_{2}, i_{3}\right)\right)$ and $q=\left(\left(i_{3}, i_{4}\right),\left(\left(i_{4}, i_{5}\right)\right.\right.$ in $\mathcal{G}(A)$ the concatenation of paths $\circ: \mathcal{G}(A) \times \mathcal{G}(A) \rightarrow \mathcal{G}(A)$ is defined as $p \circ q=\left(\left(i_{1}, i_{2}\right),\left(i_{2}, i_{3}\right),\left(i_{3}, i_{4}\right),\left(i_{4}, i_{5}\right)\right)$. The communication graph $\mathcal{G}(A)$ and powers of matrix $A$ are closely related as it is shown in the next theorem.

Theorem 23 Let $A \in \mathbb{R}_{\max }^{n \times n}$, then $\forall k \geq 1:\left[A^{\otimes k}\right]_{j i}=\max \left\{|p|_{w}: p \in P(i, j ; k)\right\}$, where $\left[A^{\otimes k}\right]_{j i}=\epsilon$ in the case when $P(i, j ; k)$ is empty i.e., no path of length $k$ from node $i$ to node $j$ exists in $\mathcal{G}(A)$.

Definition 24 Let $A \in \mathbb{R}_{\max }^{n \times n}$ then define the matrix $A^{+} \in \mathbb{R}_{\max }^{n \times n}$ as: $A^{+}=\bigoplus_{k=1}^{\infty} A^{\otimes k}$. Where the element $\left[A^{+}\right]_{j i}$ gives the maximal weight of any path from $j$ to $i$. If in addition one wants to add the possibility of staying at a node then one must include matrix $E$ in the definition of matrix $A^{+}$giving rise to its Kleene star representation defined by: $A^{*}=\bigoplus_{k=0}^{\infty} A^{\otimes k}$.

Lemma 25 Let $A \in \mathbb{R}_{\max }^{n \times n}$ be such that any circuit in $\mathcal{G}(A)$ has average circuit weight less than or equal to $\epsilon$. Then it holds that: $A^{*}=\bigoplus_{k=0}^{n-1} A^{\otimes k}$.

Definition 26 Let $G=(\mathcal{N}, \mathcal{D})$ be a graph and $i, j \in \mathcal{N}$, node $j$ is reachable from node $i$, denoted as $i \mathcal{R} j$, if there exists a path from $i$ to $j$. A graph $G$ is said to be strongly connected if $\forall i, j \in \mathcal{N}, j \mathcal{R} i$. A matrix $A \in \mathbb{R}_{\max }^{n \times n}$ is called irreducible if its communication graph is strongly connected, when this is not the case matrix $A$ is called reducible.

Remark 27 In this paper irreducible matrices are just considered. It is possible to treat the reducible case by transforming it into its normal form and computing its generalized eigenmode see [5].

\section{Spectral Theory and Linear Equations}

Definition 28 Let $A \in \mathbb{R}_{\max }^{n \times n}$ be a matrix. If $\mu \in R_{\max }$ is a scalar and $v \in R_{\max }^{n}$ is a vector that contains at least one finite element such that: $A \otimes v=\mu \otimes v$ then, $\mu$ is called an eigenvalue and $v$ an eigenvector.

Let $\mathcal{C}(A)$ denote the set of all elementary circuits in $\mathcal{G}(A)$ and write: $\lambda=\max _{p \in \mathcal{C}(A)} \frac{|p|_{w}}{|p|_{1}}$ for the maximal average circuit weight. Notice that since $\mathcal{C}(A)$ is a finite set, the maximum is attained (which is always the case when matrix $A$ is irreducible). In case $\mathcal{C}(A)=\varnothing$ define $\lambda=\epsilon$.

Definition 29 A circuit $p \in G(A)$ is said to be critical if its average weight is maximal. The critical graph of $A$, denoted by $G^{c}(A)=\left(\mathcal{N}^{c}(A), \mathcal{D}^{c}(A)\right)$, is the graph consisting of those nodes and arcs that belong to critical circuits in $G(A)$.

Theorem 30 If $A \in \mathbb{R}_{\max }^{n \times n}$ is irreducible, then there exists one and only one finite eigenvalue (with possible several eigenvectors). This eigenvalue is equal to the maximal average weight of circuits in $G(A)$ $\lambda(A)=\max _{p \in \mathcal{C}(A)} \frac{|p|_{w}}{|p|_{1}}$.

Theorem 31 Let $A \in \mathbb{R}_{\max }^{n \times n}$ and $b \in \mathbb{R}_{\max }^{n}$. If the communication graph $G(A)$ has maximal average circuit weight less than or equal to $e$, then $x=A^{*} \otimes b$ solves the equation $x=(A \otimes x) \oplus b$. Moreover, if the circuit weights in $G(a)$ are negative then, the solution is unique.

\subsection{Max-Plus Recurrence Equations for Timed Event Petri Nets}

Definition 32 Let $A_{m} \in \mathbb{R}_{\max }^{n \times n}$ for $0 \leq m \leq M$ and $x(m) \in \mathbb{R}_{\max }^{n}$ for $-M \leq m \leq-1 ; \quad M \geq 0$. Then, the recurrence equation: $x(k)=\bigoplus_{m=0}^{M} A_{m} \otimes x(k-m) ; k \geq 0$ is called an Mth order recurrence equation. 
Theorem 33 The Mth order recurrence equation, given by equation $x(k)=\bigoplus_{m=0}^{M} A_{m} \otimes x(k-m) ; k \geq 0$, can be transformed into a first order recurrence equation $x(k+1)=A \otimes x(k) ; k \geq 0$ provided that $A_{0}$ has circuit weights less than or equal to zero.

With any timed event Petri net, matrices $A_{0}, A_{1}, \ldots, A_{M} \in \mathbb{N}^{n} \times \mathbb{N}^{n}$ can be defined by setting $\left[A_{m}\right]_{j l}=a_{j l}$, where $a_{j l}$ is the largest of the holding times with respect to all places between transitions $t_{l}$ and $t_{j}$ with $m$ tokens, for $m=0,1, \ldots, M$, with $M$ equal to the maximum number of tokens with respect to all places. Let $x_{i}(k)$ denote the $k$ th time that transition $t_{i}$ fires, then the vector $x(k)=\left(x_{1}(k), x_{2}(k), \ldots x_{m}(k)\right)^{T}$, called the state of the system, satisfies the Mth order recurrence equation: $x(k)=\bigoplus_{m=0}^{M} A_{m} \otimes x(k-m) ; k \geq 0$. Now, assuming that all the hypothesis of theorem (33) are satisfied, and setting $\hat{x}(k)=\left(x^{T}(k), x^{T}(k-1), \ldots, x^{T}(k-M+1)\right)^{T}$, equation $x(k)=\bigoplus_{m=0}^{M} A_{m} \otimes x(k-m) ; k \geq 0$ can be expressed as: $\hat{x}(k+1)=\hat{A} \otimes \hat{x}(k) ; k \geq 0$, which is known as the standard autonomous equation.

\section{The Solution to the Stability Problem for Discrete Event Dynamical Systems Modeled with Timed Petri Nets}

Definition 34 A TPN is said to be stable if all the transitions fire with the same proportion i.e., if there exists $q \in \mathbb{N}$ such that

$$
\lim _{k \rightarrow \infty} \frac{x_{i}(k)}{k}=q, \forall i=1, \ldots, n
$$

This means that in order to obtain a stable TPN all the transitions have to be fired $q$ times. It will be desirable to be more precise and know exactly how many times. The answer to this question is given next.

Lemma 35 Consider the recurrence relation $x(k+1)=A \otimes x(k), k \geq 0, \quad x(0)=x_{0} \in \mathbb{R}^{n}$ arbitrary. A an irreducible matrix and $\lambda \in \mathbb{R}$ its eigenvalue then,

$$
\lim _{k \rightarrow \infty} \frac{x_{i}(k)}{k}=\lambda, \forall i=1, \ldots, n
$$

Proof. Let $v$ be an eigenvector of $A$ such that $x_{0}=v$ then,

$$
x(k)=\lambda^{\otimes k} \otimes v \Rightarrow x(k)=k \lambda+v \Rightarrow \frac{x(k)}{k}=\lambda+\frac{v}{k} \Rightarrow \lim _{k \rightarrow \infty} \frac{x_{i}(k)}{k}=\lambda
$$

Now starting with an unstable $T P N$, collecting the results given by: proposition (13), what has just been discussed about recurrence equations for TPN at the end of subsection (3.3) and the previous lemma (35) plus theorem (30), the solution to the problem is obtained.

\section{Conclusion}

The main objective of the proposal is to make it knowledgeable to large audiences. This paper gives a complete and precise solution to the stabilization problem for discrete event systems modeled with timed Petri nets combining Lyapunov theory with max-plus algebra. The presented methodology results to be innovative.

\section{References}

[1] Murata, T. (1989) Petri Nets: Properties, Analysis, and Applications. Proceedings of the IEEE, 77, 541-580. http://dx.doi.org/10.1109/5.24143

[2] Retchkiman, Z. (2005) Stability Theory for a Class of Dynamical Systems Modeled with Petri Nets. International Journal of Hybrid Systems, Vol. 4, No. 1.

[3] Lakshmikantham, V., Matrosov, V.M. and Sivasundaram, S. (1991) Vector Lyapunov Functions and Stability Analysis of Nonlinear Systems. Kluwer Academic Publ., Dordrecht. 
[4] Retchkiman, Z. (1999) From Stability to the Stabilization problem of Discrete event Systems modeled by Petri Nets. American Control Conference '99, San Diego, Cal, June 1999.

[5] Heidergott, B., Olsder, G.J. and van der Woude, J. (2006) Max Plus at Work. Princeton University Press.

[6] Baccelli, F., Cohen, G., Olsder, G.J. and Quadrat, J.P. (2001) Synchronization and Linearity. Web-Edition. 